Computing and Informatics, Vol. 39, 2020, 1, 4, doi: 10.31577/cai_2020_1-2_1

\title{
A PREFACE TO THE SPECIAL ISSUE ON EMERGING AND INTELLIGENT INFORMATION SERVICES
}

\author{
Maozhen LI \\ Department of Electronic and Computer Engineering \\ Brunel University London, Uxbridge, UB8 3PH, UK
}

\section{Zhijun DiNG}

Department of Computer Science and Technology

Tongji University, Shanghai, China

Information services have evolved from centralized monolithic systems to distributed and intelligent systems especially empowered with the emerging technologies such as big data, artificial intelligence, Internet of Things (IoTs). Papers included in this special issue mainly cover the topics of Petri net, cloud and fog computing, social networks, and deep neural networks. The following sections briefly introduce these papers.

Workflow has been playing an increasing role in modern information systems. Xiang and Liu [9] employed WFD-net, a special form of Petri net, to build a guarddriven reachability graph for correctness verification of data flows dealing with the cases that data might be missing, inconsistent, lost and redundant. According to Feng et al. [10, there is an increasing demand for business intelligence automatically extracted from event logs and process mining plays a critical role in business process management. This paper presents a new Petri net alignment to improve the efficiency in process mining. In [11], Cheng et al. presented a comprehensive learning particle swarm optimization algorithm based on fuzzy Petri net and applied it to diagnose the faults of a complex motor system. According to Li et al. [8], there is a growing research interest in imbuing robots not only with the capability of perception and planning but also the capability of learning. This paper models the behaviours of a robot into a Petri net which facilitates the learning capability of the robot. In [12], Teng et al. modelled processing mining with a logic Petri net with an aim to deal with structures which might be incomplete and concurrent to better reflect the reality of business processes. 
The past few years have witnessed the emerging of edge computing in support of IoT applications close to the edge users. Li et al. [1] presented a novel computing platform which incorporates fog computing with cloud computing in support of healthcare services. He et al. 2] employed a stochastic Petri net to model resource scheduling in cloud data centers with an aim to improve energy efficiency. A single cloud-based data storage potentially suffers from data unavailability, vendor lock-in and data privacy leakage. In [3], Wang et al. focused on multi-cloud stage with an aim to minimize monetary cost and maximize data availability. Resource virtualization plays a critical role in resource provisioning in cloud computing platforms. In [4], Pang et al. presented an improved multi-objective particle swarm optimization algorithm to optimize virtual machine deployment.

In [5], Zhang et al. considered the strength of user relationship, the similarity of entities, and the degree of user interest in recommendation systems. Interestingly, user relationship can be inferred from user interactions on social networks. In [6] Pang et al. focused on recommendation systems, and introduced stability variables and time-sensitive factors to solve the problem of user interest drift, and improve the accuracy of prediction. With a penetration of social networks, it is highly important to detect malicious information which can be hidden in data flows. In this work, Yuan and Sun [7] employed a divide and conquer strategy and proposed an intervention algorithm based on subgraph partitioning to search for influential nodes to block or release clarification.

In [13], Zhu et al. employed the Long Short-Term Memory (LSTM) deep neural network model to learn user travel patterns. The LSTM was further optimized with Quantum Genetic Algorithm (QGA). In [14], Wang et al. proposed the deep convolution and correlated manifold embedded distribution alignment (DC-CMEDA) model, which is able to realize the transfer learning classification between and among various small datasets, and greatly shorten the training time in forest fire smoke prediction. With an increasing use of mobile devices such as mobile phones, Wang et al. 15. focused on mobile sensing and proposed a particle swarm optimization algorithm for pedestrian step tracking.

We hope that the perspectives presented in this special issue would be of a great interest to the readers. We also expect the readers to contribute to this exciting and fast-growing research area.

\section{Acknowledgements}

We would like to thank Dr. Ladislav Hluchy, the Editor-in-Chief of Computing and Informatics (CAI) for his timely advice on this special issue. A big thanks also goes to Ms Viera Jablonska, the CAI journal editorial assistant for her great support in publication of the special issue. 


\section{REFERENCES}

[1] Li, Z.-Wen, L.-LiU, J.-JiA, Q.-Che, C.-Shi, C.—CAI, H.: Fog and Cloud Computing Assisted IoT Model Based Personal Emergency Monitoring and Diseases Prediction Services. Computing and Informatics, Vol. 39, 2020, No. 1-2, pp. 5-27, doi: $10.31577 /$ cai_2020_1-2_5.

[2] He, H.-Zhao, Y.-PAng, S.: Stochastic Modeling and Performance Analysis of Energy-Aware Cloud Data Center Based on Dynamic Scalable Stochastic Petri Net. Computing and Informatics, Vol. 39, 2020, No. 1-2, pp. 28-50, doi: 10.31577/cai_2020_1-2_28.

[3] Wang, P.-Zhao, C.-Liu, W.-Chen, Z.-Zhang, Z.: Optimizing Data Placement for Cost Effective and High Available Multi-Cloud Storage. Computing and Informatics, Vol. 39, 2020, No. 1-2, pp. 51-82, doi: 10.31577/cai_2020_1-2_51.

[4] Pang, S.-Dong, D.-WAng, S.: Virtual Machine Deployment Strategy Based on Improved PSO in Cloud Computing. Computing and Informatics, Vol. 39, 2020, No. 1-2, pp. 83-104, doi: 10.31577/cai_2020_1-2_83.

[5] Zhang, B.-YA Zhang, Bai, Y.-Lian, J.-Li, M.: A Multi-Dimensional Recommendation Scheme for Social Networks Considering a User Relationship Strength Perspective. Computing and Informatics, Vol. 39, 2020, No. 1-2, pp. 105-140, doi: 10.31577/cai_2020_1-2_105.

[6] Pang, S.-Yu, S.-Li, G.-QiaO, S.-Wang, M.: A Time-Sensitive Collaborative Filtering Algorithm with Feature Stability. Computing and Informatics, Vol. 39, 2020, No. 1-2, pp. 141-155, doi: 10.31577/cai_2020_1-2_141.

[7] YuAn, D.-Sun, H.: Reverse Intervention for Dealing with Malicious Information in Online Social Networks. Computing and Informatics, Vol. 39, 2020, No. 1-2, pp. 156-173, doi: $10.31577 /$ cai_2020_1-2_156

[8] Li, J.-YAng, R.-Ding, Z.-PAn, M.: A Method for Learning a Petri Net Model Based on Region Theory. Computing and Informatics, Vol. 39, 2020, No. 1-2, pp. 174-192, doi: $10.31577 /$ cai_2020_1-2_174.

[9] Xiang, D.-Liu, G.: Checking Data-Flow Errors Based on the Guard-Driven Reachability Graph of WFD-Net. Computing and Informatics, Vol. 39, 2020, No. 1-2, pp. 193-212, doi: $10.31577 /$ cai_2020_1-2_193.

[10] Feng, X.-Han, D.-Tian, Y.: Analysis and Application of Min-Cost Transition Systems to Business Process Management. Computing and Informatics, Vol. 39, 2020, No. 1-2, pp. 213-245, doi: $10.31577 /$ cai_2020_1-2_213.

[11] Cheng, X.-Wang, C.-Li, J.-Bai, X.: Adaptive Fault Diagnosis of Motors Using Comprehensive Learning Particle Swarm Optimizer with Fuzzy Petri Net. Computing and Informatics, Vol. 39, 2020, No. 1-2, pp. 246-263, doi: 10.31577/cai_2020_1$2 \_246$

[12] Teng, Y.-Du, Y.-QI, L.: A Logic Petri-Net Based Repair Method of Process Models with Incomplete Choice and Concurrent Structures. Computing and Informatics, Vol. 39, 2020, No. 1-2, pp. 264-297, doi: 10.31577/cai_2020_1-2_264. 
[13] Zhu, S.-Sun, H.-Duan, Y.-Dai, X.-SAha, S.: Travel Mode Recognition from GPS Data Based on LSTM. Computing and Informatics, Vol. 39, 2020, No. 1-2, pp. 298-317, doi: 10.31577/cai_2020_1-2_298.

[14] Wang, Y.-Liu, X.-Li, M.-Di, W.-Wang, L.: Deep Convolution and Correlated Manifold Embedded Distribution Alignment for Forest Fire Smoke Prediction. Computing and Informatics, Vol. 39, 2020, No. 1-2, pp. 318-339, doi: 10.31577/cai_2020_1-2_318.

[15] Wang, W.-Wang, C.-Wang, Z.-ZhaO, X.: An Improved PDR Localization Algorithm Based on Particle Filter. Computing and Informatics, Vol. 39, 2020, No. 1-2, pp. 340-360, doi: 10.31577/cai_2020_1-2_340

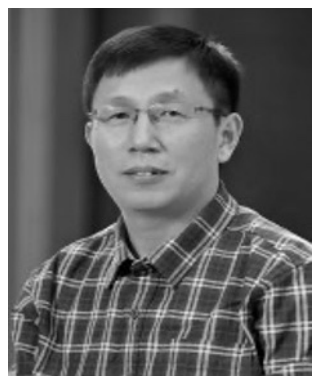

Maozhen LI is Professor in the Department of Electronic and Computer Engineering, Brunel University London, UK. He is also Visiting Professor of Tongji University, Shanghai, China. He received his Ph.D. from the Institute of Software, Chinese Academy of Sciences in 1997. His main research interests include high performance computing, big data analytics and intelligent systems with applications to smart grid, smart manufacturing and smart cities. He has over 180 research publications in these areas including 4 books. He has served over 30 IEEE conferences and is on the editorial board of a number of journals including journal of Computing and Informatics. He is Fellow of the British Computer Society (BCS) and the Institute of Engineering and Technology (IET).

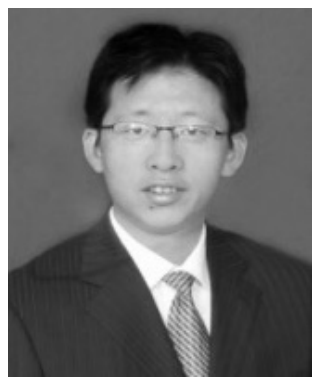

Zhijun DiNG is currently Professor with the Department of Computer Science and Technology, Tongji University, Shanghai, China. He received the M.Sc. degree from the Shandong University of Science and Technology, Tai'an, China, in 2001, and the Ph.D. degree from Tongji University, Shanghai, China, in 2007. He has published over 100 papers in domestic and international academic journals and conference proceedings. His research interests are in formal engineering, Petri nets, services computing, and mobile internet. He is a Senior Member of IEEE since 2015. 